JGG 2020;68:235-239

doi: 10.36150/2499-6564-282

\title{
Neurological features of COVID-19 infection: a case series of geriatric patients
}

\author{
Francesca Crosta ${ }^{1,2}$, Paola Giustina Simeone ${ }^{2,3}$, Carlo Sanrocco ${ }^{4}$, \\ Pasquale Lanzilotta ${ }^{1}$, Antonella Cecamore ${ }^{1}$, Franco Colameco ${ }^{1}$, \\ Giovambattista Desideri², Francesca Santilli ${ }^{3}$, Rosa Scurti ${ }^{1}$, \\ Giustino Parruti ${ }^{5}$ \\ ${ }^{1}$ Department of Geriatric Unit, Azienda Sanitaria Locale (AUSL) di Pescara, Italy; ${ }^{2}$ Department of \\ Life, Health \& Environmental Sciences, University of L'Aquila, Coppito-L'Aquila, Italy; ${ }^{3}$ Department \\ of Medicine and Aging, Center for Advanced Studies and Technology (CAST), University "G. \\ D’Annunzio" of Chieti-Pescara, Chieti, Italy; ${ }^{4}$ Department of Internal Medicine, Azienda Sanitaria \\ Locale (AUSL) di Pescara, Italy, ${ }^{5}$ Department of Infectious Disease, Azienda Sanitaria Locale (AUSL) \\ di Pescara, Italy
}

The coronavirus SARS-CoV-2 (severe acute respiratory syndrome coronavirus 2), which is responsible for the coronavirus disease 2019 (COVID-19), is known to cause substantial pulmonary disease. However, clinicians observed many extrapulmonary manifestations of COVID-19. Neurological manifestations are being recognised increasingly. On the basis of knowledge of other coronaviruses, especially those that caused the severe acute respiratory syndrome and Middle East respiratory syndrome epidemics, cases of central nervous system disease caused by SARS-CoV-2 might be expected to be rare.

We report four cases of neurologic manifestations of SARS-CoV-2 in elderly patients who presented to the Geriatric Department of Pescara Hospital (Italy).

Key words: SARS-CoV-2, neurological symptoms, systemic inflammation, vascular injury

\section{CASE-1-STROKE}

A 83-year-old man had reduced level of consciousness, dysphasia, hemiplegia on right side, dysarthria, sensory deficit over a period of 36 hours.

The medical history was negative for the major cardiovascular risk factors. When he presented to the hospital, the score on the National Institutes of Health Stroke Scale (NIHSS) was 13 (scores range from 0 to 42, with higher numbers indicating greater stroke severity) ${ }^{1}$, and computed tomography $(C T)$ and CT angiography of the cerebral arteries showed a infarction of the left middle cerebral artery with a partially occlusive thrombus in the left carotid artery at the cervical bifurcation and occlusion of the left middle artery (image A). Blood test showed D-dimer 52 ng/ml, fibrinogen 370 mg/ $\mathrm{dl}, 9900$ white-cell count per $\mathrm{mm}^{3}$ with lymphopenia. Electrocardiogram showed sinus rhythm. Antiplatelet therapy was started (aspirin $100 \mathrm{mg}$ daily). Stroke workup with echocardiography and carotid ultrasound of the head and neck did not reveal the source of the thrombus.

One day later, the patient developed pyrexia $\left(38.2^{\circ} \mathrm{C}\right)$, dyspnoea and

This is an open access article distributed in accor (he CC-BY-NC-ND (Creative Commons Attribution-NonCommercial-NoDerivatives 4.0 International) license. The article can be used by giving appropriate credit and mentioning the license, but only for non-commercial purposes and only in the original version. For further information: https://creativecommons.org/licenses/by-nc-nd/4.0/deed.en 
cough. High-resolution chest CT was performed and it showed patchy ground-glass opacities in bilateral lung apices.

The oropharyngeal and nasopharyngeal swabs for SARS-CoV-2 turned out positive.

The patient was treated with hydroxychloroquine 200 mg BID, clarithromycin 500 mg bid, high dose corticosteroids (dexamethasone 20 mg/die) and heparin (4000 UI/die) ${ }^{2,3}$.

Repeat CT angiography on hospital day 10 showed complete resolution of the thrombus, and the patient was discharged to a rehabilitation facility with an almost complete resolution of the neurological findings.

\section{CASE 2-DELIRIUM}

A 76-year-old man was admitted in hospital for drowsiness suggestive for hyperactive delirium. At the rapid clinical test for delirium assessment (4AT), the patient presented a score of 5 suggestive of possible delirium ${ }^{4}$. Medical history includes hypertension and osteoarthritis. He was driving and living independently, with help from a visiting nurse, but with some known cognitive impairment.

On arrival, neurology consultant observed right greater than left cogwheel rigidity and myoclonus (left greater than right). Electroencephalogram was performed and it showed diffuse theta slowing (image B), frequent and low amplitude isolated generalized discharges, and frequent brief periods of $1 \mathrm{~Hz}$ generalized rhythmic delta activity with sharp contouring and bilateral frontal predominance. He never presented respiratory symptoms or hemodynamic instability beyond a brief fever to $37.8^{\circ} \mathrm{C}$. He had a persistently elevated C-reactive protein, with a maximum of $130 \mathrm{mg} / \mathrm{L}$, LDH $210 \mathrm{mg} / \mathrm{dL}$, lymphocytopoenia and creatinine $1.88 \mathrm{mg} / \mathrm{dl}$.

He was noted to have a slight cough prompting SARSCoV-2 polymerase chain reaction (PCR) testing, which returned positive.

The patient was treated with high dose corticosteroids (dexamethasone $20 \mathrm{mg} / \mathrm{die}$ ) and heparin (4000 Ul/die) 2,3. The patient died two days after the admission to our department for sudden cardiac death.

The autopsy revealed the presence of thrombi and inflammatory infiltrates in the wall of coronary arteries and pulmonary circulation mimicking pulmonary embolism and vasculitis respectively.

\section{CASE 3-ENCEPHALITIS}

A 79-year-old male with a history of hypertension, diabetes and chronic heart failure was admitted to our department for altered state of consciousness and hyperpyrexia $\left(\mathrm{TC} 39^{\circ} \mathrm{C}\right)$ started the day before the hospitalization. He was moderately frail (Clinical Frailty Scale 6) ${ }^{5}$ and he lived alone.

The patient was disorientated in time and space and he had concentration and attention difficulties. Bedside cognitive testing revealed immediate and short-term memory deficits. The patient mentioned a loss of smell and taste. He was anxious. Somatic fixations were noted.

A diagnostic assessment was initiated including a CT scan of the brain, which was normal.

Electroencephalogram showed a general excess of beta-rhythm, as seen with benzodiazepine treatment as well as infrequent, short-lasting rhythmic left temporal delta activity, never exceeding $10 \mathrm{~s}$. The day after admission, a brain magnetic resonance imaging (MRI) showed an asymmetric FLAIR (Fluid-attenuated inversion recovery) hyperintensity of the left medial temporal cortex (image C) associated with mild gyral expansion without any diffusion restriction or contrast enhancement.

Lumbar puncture was performed, and the analysis of cerebrospinal fluid showed white blood cells $\left(0.001 \times 10^{9} / \mathrm{L}\right)$, protein $(0.27 \mathrm{~g} / \mathrm{L})$, adenosine deaminase $(0.17 \mathrm{U} / \mathrm{L})$ and sugar $(3.14 \mathrm{mmol} / \mathrm{L})$ contents within normal limits. Further research to rule out infectious, paraneoplastic and auto-immune causes of encephalitis was performed, including a CT scan of the abdomen and chest, a comprehensive auto-immune screening, serologic testing, detection of anti-neuronal antibodies on blood. No infectious or alternative causes for encephalitis have been demonstrated.

Given the persistence of high fever and the onset of dyspnoea PCR testing for SARS-CoV-2 was performed and it resulted positive. Therapy with clarithromycin $500 \mathrm{mg}$ bid, high dose corticosteroids (dexamethasone $20 \mathrm{mg} / \mathrm{die}$ ) and heparin (4000 Ul/die) was started 2,3.

In the following days, cognitive performance improved. Four days later, a second brain MRI was performed and it showed normalization of cortical hyperintensity and gyral expansion.

\section{CASE 4-SYNCOPE}

A 79-year-old woman with a past medical history of hypertension and chronic renal failure $\left(2^{\text {nd }}\right.$ stage according to K/DOQI guidelines for Chronic Kidney Disease) ${ }^{6}$ acceded to our department for syncope resulting in brain injury. The patient was on drug therapy with angiotensin-converting enzyme (ACE) inhibitors and amlodipine. Three days before admission, she developed myalgia and cough followed by fever up to $39^{\circ} \mathrm{C}$. 
Baseline clinical examination was significant for a temperature of $38.9^{\circ} \mathrm{C}$, oxygen saturation of $97 \%$, elevated blood pressure and tachycardia.

Laboratory exams were remarkable for lymphopenia with an absolute lymphocyte count of $0.4 \times 10^{9} / \mathrm{L}$, creatinine $4.53 \mathrm{mg} / \mathrm{dL}$, C-reactive protein $139 \mathrm{mg} / \mathrm{L}$ and D-dimer $7.4 \mathrm{ng} / \mathrm{ml}$.

Orthostatic hypotension was excluded. Electrocardiogram and echocardiogram did not reveal any arrhythmias or significant changes of structural cardiac defects respectively.

Brain CT and electroencephalogram resulted both negative.

High-resolution chest CT showed bilateral peripheral ground-glass opacities, while CT pulmonary angiogram excluded the presence of embolism. The oropharyngeal and nasopharyngeal swabs for SARS-CoV-2 returned positive.

Patient was treated with clarithromycin $500 \mathrm{mg}$ bid, high dose corticosteroids (dexamethasone $20 \mathrm{mg} / \mathrm{die}$ ) and heparin (4000 UI/die) 2,3.

After ten days, given significant improvement in symptoms and resolution of light-headedness, she was discharged at home.

\section{DISCUSSION}

A number of non-specific mild neurological symptoms have been identified in hospitalized patients with COVID-19 ${ }^{7}$. Nervous system manifestations were significantly more common in severe infections compared with nonsevere infections ${ }^{8}$ they included acute cerebrovascular disease; ischemic stroke and cerebral hemorrhage, impaired consciousness and skeletal muscle injury.

The patients with severe infection were significantly older and more likely to have other underlying disorders, especially hypertension, and had fewer typical symptoms of COVID-19 such as fever and dry cough ${ }^{9}$. Precise case definitions must be used to distinguish nonspecific complications of severe disease (eg, hypoxic encephalopathy and critical care neuropathy) from those caused directly or indirectly by the virus, including infectious, para-infectious, and post-infectious encephalitis, hypercoagulable states leading to stroke ${ }^{10,11}$.

Our case series demonstrated how recognition of neurological disease associated with SARS-CoV-2 in patients whose respiratory infection is mild or asymptomatic might prove challenging, especially if the primary COVID-19 illness occurred days earlier.

The mechanism of cerebrovascular manifestation in COVID-19 patients is complex and likely multifactorial.
In a single centre retrospective study of 221 admitted COVID-19 patients in Wuhan, Li YC et al. ${ }^{12}$ found that 13 patients (5.9\%) developed acute cerebrovascular events. Of these patients, 11 had acute ischemic stroke (5\% of admitted COVID-19 patients), one (0.5\%) had cerebral venous sinus thrombosis and one $(0.5 \%)$ had intracerebral haemorrhage.

$\mathrm{Li} \mathrm{Y}$ et al. ${ }^{12}$ found that patients with cerebrovascular manifestations with COVID-19 had significantly higher average D-dimer and $\mathrm{C}$ reactive protein levels, potentially suggesting an inflammatory-induced hypercoagulable state resulting in stroke.

Moreover, patients with pre-existing cerebrovascular disease resulting in intracranial stenosis with hypoperfused brain regions may be at increased risk for ischemic stroke while in a state of severe infection and systemic inflammation.

Early in 2002 and 2003, studies on the samples from patients with SARS have demonstrated the presence of SARS-CoV particles in the brain, where they were located almost exclusively in the neurons ${ }^{12-14}$.

Increasing evidence shows that CoVs may first invade peripheral nerve terminals, and then gain access to the CNS via a synapse-connected route ${ }^{12}$.

These mechanisms are also involved in the pathogenesis of hyperactive delirium associated with SARS-CoVs infection. Delirium may be a manifestation of direct central nervous system (CNS) invasion, induction of CNS inflammatory mediators, a secondary effect of other organ system failure, an effect of sedative strategies, prolonged mechanical ventilation time, or environmental factors, including social isolation. Indirectly mechanisms favouring delirium are represented by hypoxia, high fever, dehydration, medications or metabolic derangements.

Acute brain dysfunction, symptomatically presenting as delirium (also called encephalopathy), may be a feature of the neuro-invasive potential of SARS-CoV-2 well demonstrated during SARS and MERS epidemics ${ }^{11}$.

Encephalitis has also been described in one case of SARS-CoV-2. The possible brain entry routes for CoVs, including SARS-CoV-2, include either direct intranasal access to the brain via olfactory nerves (with anosmia as an early symptom) or indirect access to the brain by crossing the blood-brain barrier (BBB) via haematogenous or lymphatic spread. In the ENCOVID-multicentre study ${ }^{15} 25$ cases of encephalitis in SARS-CoV-2 infection were included in a prospective observational multicentre study. Encephalitis cases in COVID-19 exhibited a wide heterogeneity in terms of clinical features, CSF, $\mathrm{MRI}$ findings, response to treatment and outcomes with 13 cases with normal MRI, 7 with heterogeneous MRI alterations and rare ADEM/limbic encephalitis cases. Heterogeneity of presentation, response to treatment 

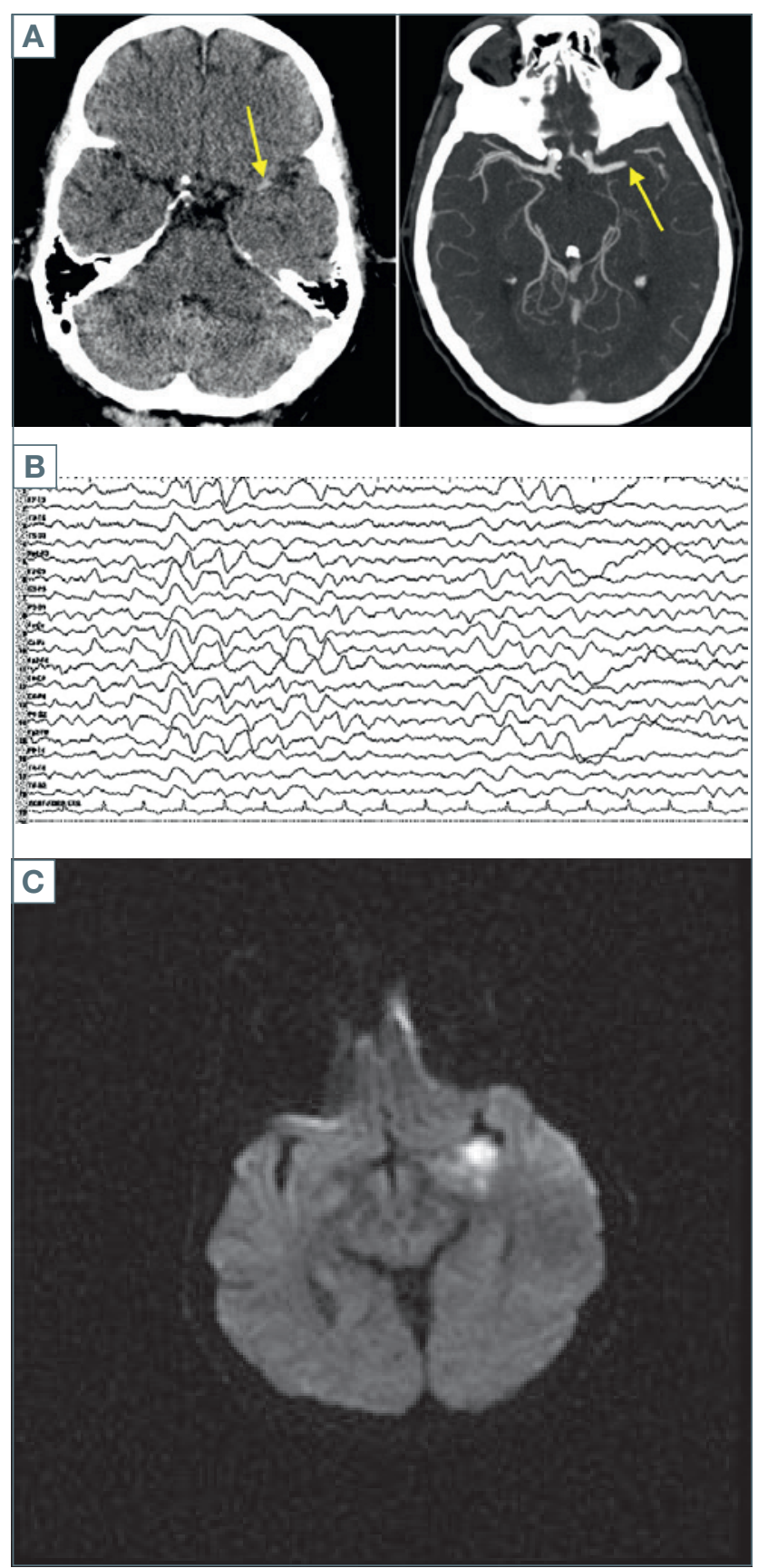

Figure 1. A) CT angiography of the cerebral arteries showed (blue line) occlusion of the left middle cerebral artery; B) Electroencephalogram showed diffuse theta slowing; $C$ ) Hyperintensity in left medial temporal lobe on diffusion weighted imaging.

and outcomes of encephalitis of COVID-19 underlines different pathophysiological mechanisms.

Finally, we reported a case of syncope as the only initial symptom of COVID-19 infection.

To our knowledge, syncope alone has rarely been described as a symptom associated with COVID-19 infection and it was in most cases secondary to structural cardiac disease or pulmonary embolism. We report a case of syncope as the presenting symptom in a patient initially asymptomatic for COVID-19 infection and in which cardiac causes where immediately ruled out.

The proposed pathophysiological explanation might be a neurally mediated/reflex mechanism in its no classical form (absence of certain trigger) or an autonomic dysfunction, either primary or secondary. There are two main pathophysiological mechanisms in reflex syncope, both of them depending on an imbalance between sympathetic and parasympathetic activity: vasodepression, caused by insufficient sympathetic vasoconstriction; and cardioinhibition, caused by parasympathetic predominance. This type of imbalance might find another confirming factor in the association between COVID-19 infection and "!inappropriate"! sinus tachycardia, which often characterizes patients with novel coronavirus infection even in the absence of respiratory failure.

The autonomic failure hypothesis, on the other hand, might involve a primary failure caused by the virus itself or a secondary failure due to autoimmune autonomic neuropathy. However, determining if this is a direct effect of the virus or if it is due to a critical autoimmune reaction or inflammation caused by cytokines is still a matter of debate. Cytokine storm with subsequent hyper-inflammation is one of the proposed mechanisms of COVID-19 serious illness. Vascular injury caused by IL-1 and IL-6 and decreased systemic vascular resistance resulting in vasodilation could be one of the possible explanations of syncope as the resulting symptom. Finally, in our case the patient was on chronic therapy with ACE-inhibitors. Whether ACE inhibitors, up-regulating ACE-2 expression in many tissues and therefore facilitating virus binding to the cells, predispose to COVID-19 infection has not been proved. However, the overall decrease in the vasopressor effect of angiotensin II in patients treated with ACE inhibitors might play a role in the pathophysiology of syncope ${ }^{16,17}$.

In the Helm's observational series of hospitalized patients with COVID-19 and neurological features, the median age of the patients was 63 years, and the median Simplified Acute Physiology Score II at the time of neurologic examination was 52 (interquartile range, 37 to 65 , on a scale ranging from 0 to 163, with higher scores indicating greater severity of illness ${ }^{17}$.

Further studies with larger sample size are necessary before drawing definite conclusions on the exact mechanisms linking neurological findings with COVID-19 infection.

Careful clinical, diagnostic, and epidemiological studies are needed to help define the manifestations and burden of neurological disease caused by SARS-CoV-2. 


\section{ACKNOWLEDGMENTS}

We are grateful to Clinical and Microbiological staff of Pescara General Hospital, particularly for assistance in diagnosis.

\section{References}

1 Pezzella FR, Picconi O, De Luca A, et al. Development of the Italian Version of the National Institutes of Health Stroke Scale: It-NIHSS. Stroke 2009;40:2557-9.

2 Sanders JM, Monogue ML, Jodlowski TZ, et al. Pharmacologic treatments for coronavirus disease 2019 (COVID-19). A review. JAMA 2020;Apr 13. https://doi.org/10.1001/ jama.2020.6019

$3 \mathrm{NIH}$ COVID-19 treatment guidelines.

4 Bellelli G, Morandi A, Davis DH, et al. Validation of the 4AT, a new instrument for rapid delirium screening: a study in 234 hospitalised older people. Age Ageing 2014;43:496-502.

5 lqbal J, Denvir M, Gunn J. Frailty assessment in elderly people. Lancet 2013;381:1985-6.

6 National Kidney Foundation. K/DOQI clinical practice guidelines for chronic kidney disease: evaluation, classification, and stratification. Archived from the original on 2005-04-15. Retrieved 2008-06-29.

7 Gupta A, Madhavan MV, Sehgal K, et al. Extrapulmonary manifestations of COVID-19. Nat Med 2020;26:1017-32.

8 Mao L, Jin H, Wang M, et al. Neurologic manifestations of hospitalized patients with coronavirus disease 2019 in Wuhan, China. JAMA Neurol 2020;77:683-90. https://doi. org/10.1001/jamaneurol.2020.1127
9 Ellul MA, Benjamin L, Singh B, et al. Neurological associations of COVID-19. Lancet Neurol 2020;S14744422(20)30221-0.

10 Bergmann CC, Lane TE, Stohlman SA. Coronavirus infection of the central nervous system: host-virus stand-off. Nat Rev Microbiol 2006;4:121-32.

11 Li YC, Bai WZ, Hashikawa T. The neuroinvasive potential of SARS-CoV-2 may play a role in the respiratory failure of COVID-19 patients. J Med Virol 2020. https://doi. org/10.1002/jmv.25728

$12 \mathrm{Li} \mathrm{Y,} \mathrm{Li} \mathrm{H,} \mathrm{Fan} \mathrm{R,} \mathrm{et} \mathrm{al.} \mathrm{Coronavirus} \mathrm{infections} \mathrm{in} \mathrm{the} \mathrm{central}$ nervous system and respiratory tract show distinct features in hospitalized children. Intervirology 2016;59:163-9.

${ }_{13} \mathrm{Xu} \mathrm{J}$, Zhong S, Liu J, et al. Detection of severe acute respiratory syndrome coronavirus in the brain: potential role of the chemokinemig in pathogenesis. Clin Infect Dis 2005;41:1089-96.

${ }_{14} \mathrm{Gu}$ J, Gong E, Zhang B, et al. Multiple organ infection and the pathogenesis of SARS. J Exp Med 2005;202:415-24.

15 Pilotto A, Masciocchi S, Volonghi I, et al. Clinical presentation and outcomes of SARS-CoV-2 related encephalitis: the ENCOVID multicentre study. J Infect Dis 2020;Sep 28:jiaa609.

16 Wiesmann M, Kiliaan AJ, Claassen JA. Vascular aspects of cognitive impairment and dementia. J Cereb Blood Flow Metab 2013;33:1696-706.

17 Helms J, Kremer S, Merdji H, et al. Neurologic features in severe SARS-CoV-2 Infection. N Engl J Med 2020;Apr 15. https://doi.org/10.1056/NEJMc2008597 